\title{
Noncentral Forces in Crystals of Charged Colloids
}

\author{
D. Reinke, ${ }^{1}$ H. Stark, ${ }^{2}$ H.-H. von Grünberg, ${ }^{3}$ Andrew B. Schofield, ${ }^{4}$ G. Maret, ${ }^{1}$ and U. Gasser ${ }^{5}$ \\ ${ }^{1}$ Physics Department, University of Konstanz, 78457 Konstanz, Germany \\ ${ }^{2}$ Max-Planck-Institut für Dynamik und Selbstorganisation, Bunsenstrasse 10, D-37073 Göttingen, Germany \\ ${ }^{3}$ Karl-Franzens-Universität, 8010 Graz, Austria \\ ${ }^{4}$ School of Physics, University of Edinburgh, Edinburgh, Scotland EH9 3JZ, United Kingdom \\ ${ }^{5}$ Laboratory for Neutron Scattering, ETH Zurich \& Paul Scherrer Institut, 5232 Villigen PSI, Switzerland
} (Received 27 July 2006; published 19 January 2007)

\begin{abstract}
The elastic properties of fcc crystals consisting of charge stabilized colloidal particles are determined from real space imaging experiments using confocal microscopy. The normal modes and the force constants of the crystal are obtained from the fluctuations of the particles around their lattice sites using the equipartition theorem. We show that the Cauchy relation is not fulfilled and that only noncentral manybody forces can account for the elastic properties.
\end{abstract}

DOI: 10.1103/PhysRevLett.98.038301

Understanding how an elastic material deforms under external mechanical stresses is essential knowledge in material science and of fundamental interest. While in metals and other hard materials a great deal is known about elasticity, its study currently receives a lot of attention in soft materials because of their great potential for the development of novel materials. The response to external stresses is described by the elastic constants $C_{\mu \nu \rho \sigma}$, which are defined as the second derivatives of the elastic free energy with respect to components of the strain tensor $\eta_{\mu \nu}$ [1]. In crystals with an fcc lattice, it follows from the cubic point symmetry that the elastic properties are described by only three independent constants: $C_{11}, C_{12}$, and $C_{44}$, where the indices are given in Voigt notation [1]. The number of independent constants is further reduced by the Cauchy relation $C_{12}=C_{44}$ if the atoms interact through central forces [2]. Whether central or noncentral forces act in a fcc crystal can, therefore, be decided by measuring macroscopic elastic constants. E.g., the Cauchy relation is not fulfilled in metals; the noncentral forces in metals are due to the conduction electrons that screen the charges of the ions and contribute to the bulk modulus but not to the shear moduli [2]. In analogy to metals, crystals of charged colloidal particles consist of large macroions in suspension that are surrounded by the much smaller microions [3]. Therefore, the question arises whether the elastic properties of colloidal crystals are comparable to metals and whether noncentral forces are needed to characterize the interactions between the macroions. In analogy to metals, where effective potentials between the ions serve to eliminate the degrees of freedom of the conduction electrons, theories of effective macroion-macroion interactions have been developed for colloids to eliminate the degrees of freedom of the microions. The most important effective interaction in colloids is the very successful DerjaguinLandau-Verwey-Overbeek interaction potential [4], which has been a cornerstone for the understanding of colloidal suspensions for the last 50 years. However, in suspensions with high colloid density and a long screening length
PACS numbers: 82.70.Dd, 05.40.-a, 62.20.Dc, 63.20.Dj

many-body forces are expected to become important that cannot be taken into account by a radially symmetric effective pair-potential [5]. Indeed, such forces have been observed in colloidal liquids [6-8] and they are expected to be important for the elasticity of colloidal crystals [9]. But the internal forces and all elastic moduli of threedimensional (3D) colloidal single crystals have never been measured before.

Here, we present 3D real space imaging data of colloidal monocrystals with fcc symmetry that allow to extract the force constants (FCs) of the crystal from the dispersion relations of overdamped normal modes and, in the $q \rightarrow 0$ limit, all elastic constants of the crystal. Moreover, our results show clearly that the interaction between the colloidal particles in the crystal cannot be described in terms of central forces. As a major advantage over dynamic light scattering experiments [10] hydrodynamics does not play any role for our analysis. Our methods only rely on "snapshots" of the particle positions that are used to calculate ensemble averages and, therefore, the frictional forces between the colloid particles and the solvent never enter our analysis.

We used poly-methylmethacrylate spheres [11] with a diameter $\sigma=1.66 \mu \mathrm{m}$ and a polydispersity of $<5 \%$ that were sterically stabilized, fluorescently labeled with rhodamine, and suspended in a mixture of decahydronaphtalene and cycloheptylbromide that matches both the density and the refractive index of the particles. Because of the careful density matching with a precision of $\sim 10^{-3} \mathrm{~g} / \mathrm{cm}^{3}$, sedimentation of the particles was found to be negligible in all measurements. At the same time, the refractive index of the solvent was close to that of the particles, and it was possible to take images at a depth of up to $70 \mu \mathrm{m}$ from the cover slip. The particles were observed with a fast Nipkow-disk laser-scanning confocal microscope using a CCD camera. The resolution in the image plane was $\approx 220 \mathrm{~nm}$ and $\approx 770 \mathrm{~nm}$ in the direction of the optical axis. A $100 \times$ objective lens (numerical aperture 1.45) was used to observe a volume $V \approx 58 \mu \mathrm{m} \times 55 \mu \mathrm{m} \times 20 \mu \mathrm{m}$ 
inside the much larger sample cell. The objective was mounted on a piezo translation stage for the scanning in $z$ direction. The distance from the cover slip to the observed volume was never smaller than $20 \mu \mathrm{m}$ in order to avoid wall effects of the cover slip. Typically, 5000 particles were observed in one 3D snapshot. The frame rate was 43 images/s, and the spacing between images was $\Delta z=$ $0.3 \mu \mathrm{m}$. It took $\approx 1.5 \mathrm{~s}$ to observe the whole volume $V$ and, consequently, the measured particle coordinates are not strictly snapshots of a particle configuration. DerjaguinLandau-Verwey-Overbeek interaction potential (DLVO). The particles are detected by looking for local intensity maxima and their positions can be determined with a subpixel accuracy of $\approx 20 \mathrm{~nm}$, since one particle comprises a large number of volumetric pixels [12]. Samples with volume fractions $0.01<\phi<0.41$ were prepared in order to map out the phase behavior; all measurements were carried out at $T=295 \mathrm{~K}$. Freezing was observed in samples with volume fractions larger than $\phi_{f} \approx 0.31$. The phase behavior compares well with that of hard spheres with Yukawa repulsion (HSY) with the interaction potential $V(r)=V_{\mathrm{HS}}(r)+\frac{B \sigma}{r} \exp (-\kappa[r-\sigma])$ [13]. Here, $V_{\mathrm{HS}}(r)$ is the hard sphere potential, $\kappa$ is the inverse Debye screening length, and $B=k_{B} T \frac{\lambda_{B}}{\sigma}\left(\frac{Z_{\text {eff }}}{1+\kappa \sigma / 2}\right)^{2}$ is the contact value with the Bjerrum length $\lambda_{B}$ and the charge number $Z_{\text {eff }}$. By comparing measured radial distribution functions $g(r)$ from liquid samples with those from Monte Carlo (MC) simulations of HSY particles, we find $\kappa^{-1}=221 \pm 30 \mathrm{~nm}$ and $Z_{\text {eff }}=245 \pm 40$. These values are in reasonable agreement with a recent study of the interactions in a similar colloidal dispersion [14].

For $\phi \geq 0.31$ large random hexagonal close packed crystals [15] with hexagonal planes oriented parallel to the cover slip formed within several days, and we chose regions of three to ten hexagonal layers with fcc stacking for our measurements. The lattice constant $a$ of the Bravais unit cell was measured, and the structure of these crystals was compared with a perfect fcc lattice. The deviations of the average particle positions from the fcc-lattice positions are small. For the calculation of the deviation $\boldsymbol{u}_{i}(t)=$ $\boldsymbol{r}_{i}(t)-\boldsymbol{R}_{i}$ of particle $i$, the reference position $\boldsymbol{R}_{i}$ is determined by averaging its position over all snapshots taken during the measurement. The duration of a measurement was chosen such that it contains $\gtrsim 100$ statistically independent particle configurations. As shown in Fig. 1(a), the distributions of the components $u_{\mu}$ of the deviations are Gaussian at all volume fractions, so we are always in the harmonic regime of crystal elasticity. The component parallel to the optical axis of the microscope (not shown) has a broader distribution due to the lower resolution in this direction. Since at all $\phi$ the width of the distribution is about $1 / 4$ of the surface-to-surface distance of nearest neighbor particles, the influence of the HS part of the potential is small and the interactions are dominated by electrostatic forces. Following essentially the same procedure as in Ref. [16], the dynamical matrix $D_{\mu \nu}(\boldsymbol{q})$
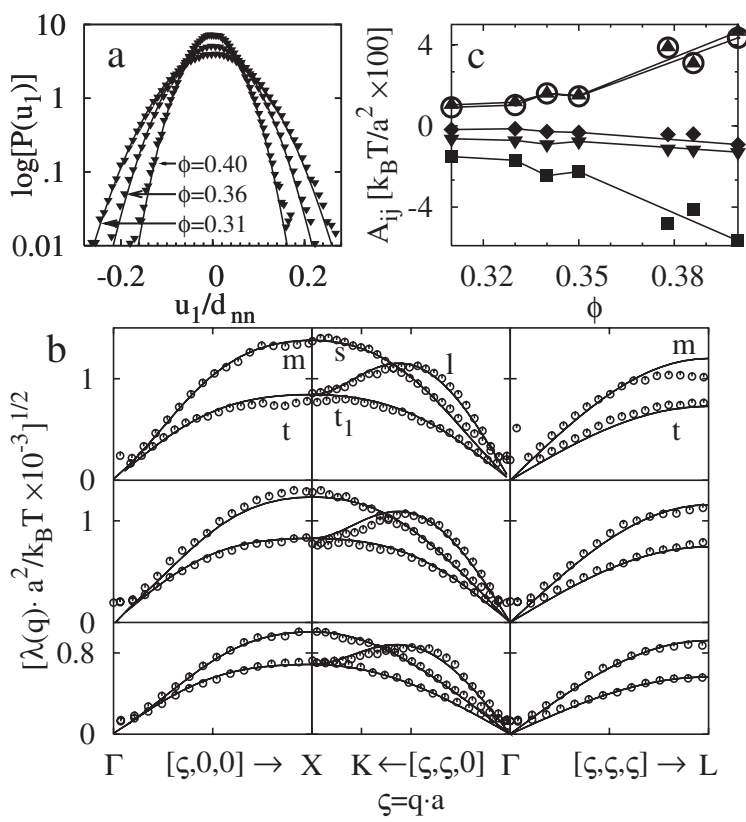

FIG. 1. (a) Distributions of the deviations $u_{1}$ normalized to the nearest neighbor distance $d_{n n}$ at several $\phi$. The distributions of $u_{2}$ coincide with the ones for $u_{1}$. The lines represent Gaussian fits to the data. (b) Measured dispersion relations are shown for (top to bottom) $\phi=0.38,0.34$, and 0.31 together with fits to the data. $K, X$, and $L$ denote the edge of the first Brillouin zone in (1, $1,0),(1,0,0)$, and $(1,1,1)$ direction, respectively. For each column, the modes are identified in the topmost panel. See text for details. (c) FCs obtained from the fits in (b). $A_{11}^{(1)}$ (squares), $A_{12}^{(1)}$ (down triangles), $A_{33}^{(1)}$ (open circles), $A_{11}^{(2)}$ (up triangles), and $A_{22}^{(2)}$ (diamonds); errors are comparable to the size of the symbols. The lines are guides to the eye.

[2] is determined using the equipartition theorem; each $\boldsymbol{q}$ mode of the harmonic approximation $U=$ $\frac{1}{2} \sum_{q, \mu, \nu} u_{\mu}(\boldsymbol{q}) D_{\mu \nu}(\boldsymbol{q}) u_{\nu}^{*}(\boldsymbol{q})$ to the elastic energy of the crystal contains the thermal energy $k_{B} T / 2$. Therefore, the inverse of the dynamical matrix can be obtained from an ensemble average of the measured particle deviations:

$$
D_{\mu \nu}^{-1}(\boldsymbol{q})=\frac{\left\langle u_{\mu}(\boldsymbol{q}) u_{\nu}^{*}(\boldsymbol{q})\right\rangle}{k_{B} T} .
$$

We calculate the ensemble average $\langle\ldots\rangle$ by taking a time average over all measured configurations and we analyze modes with wave vectors $\boldsymbol{q} \propto(1,1,0),(1,1,1)$, and $(1,0$, $0)$. Since these directions in reciprocal space correspond to symmetry axes of two-, three-, or fourfold rotations, the eigenmodes in these directions are a longitudinal mode $l$ and two transverse modes $t_{1}$ and $t_{2}$ with eigenvectors $\hat{\boldsymbol{e}}_{l}$, $\hat{\boldsymbol{e}}_{t_{1}}$, and $\hat{\boldsymbol{e}}_{t_{2}}$, respectively. To circumvent the problem of the lower precision of the particle coordinates parallel to the optical axis, we determine the properties of the crystal by using only the components of $\boldsymbol{u}(\boldsymbol{q})$ that are perpendicular to the optical axis, which coincides with the $(1,1,1)$ direction. These are the components $u_{p}(\boldsymbol{q})=\frac{1}{\sqrt{N}} \sum_{\boldsymbol{R}} \boldsymbol{u}(\boldsymbol{R}) \cdot$ $\hat{\boldsymbol{p}} \exp (i \boldsymbol{R} \cdot \boldsymbol{q})$ with $\hat{\boldsymbol{p}}=\frac{1}{\sqrt{2}}(1,-1,0)$ or $\frac{1}{\sqrt{6}}(-1,-1,2)$ and 
equivalent directions that are obtained by threefold rotations around $(1,1,1)$. As $\boldsymbol{q}$ enters only in combination with the lattice points $\boldsymbol{R}$ that are known with high accuracy, there is no limitation for the choice of $\boldsymbol{q}$. If the polarization $\hat{\boldsymbol{p}}$ is an eigenvector of the dynamical matrix $\boldsymbol{D}(\boldsymbol{q})$, then $\left\langle\left|u_{p}(\boldsymbol{q})\right|^{2}\right\rangle$ yields the eigenvalue $\lambda_{p}(\boldsymbol{q}):=k_{B} T /\left\langle\left|u_{p}(\boldsymbol{q})\right|^{2}\right\rangle$ of the dynamical matrix, e.g., if $\hat{\boldsymbol{q}}=\hat{\boldsymbol{p}}=\frac{1}{\sqrt{2}}(1,-1,0)$. Otherwise, we express $\hat{p}$ in terms of the eigenvectors and $\left\langle\left|u_{p}(\boldsymbol{q})\right|^{2}\right\rangle$ is given by a mixture of eigenvalues. E.g., for $\hat{\boldsymbol{p}}=\frac{1}{\sqrt{6}}(-1,-1,2)$ and $\hat{\boldsymbol{q}}=\frac{1}{\sqrt{2}}(1,-1,0)$ we obtain $\hat{\boldsymbol{p}}=$ $\frac{1}{\sqrt{3}} \hat{\boldsymbol{e}}_{t_{1}}+\frac{2}{\sqrt{6}} \hat{\boldsymbol{e}}_{t_{2}}$ and the measured quantity $\lambda_{s}(\boldsymbol{q}):=$ $k_{B} T /\left\langle\left|u_{p}(\boldsymbol{q})\right|^{2}\right\rangle$ can be expressed in terms of the eigenvalues of the dynamical matrix: $\lambda_{s}=3 \lambda_{t_{1}} \lambda_{t_{2}} /\left(2 \lambda_{t_{1}}+\lambda_{t_{2}}\right)$, where $\lambda_{t_{1}}$ and $\lambda_{t_{2}}$ are the eigenvalues corresponding to the eigenvectors $\hat{\boldsymbol{e}}_{t_{1}}=\frac{1}{\sqrt{2}}(1,1,0)$ and $\hat{\boldsymbol{e}}_{t_{2}}=(0,0,1)$. In this way we can determine the modes $l, t_{1}$, and $s$ for wave vector $\boldsymbol{q} \propto(1,1,0)$ and equivalent directions, $t_{1}$ and $m$ for $\boldsymbol{q} \propto(1,0,0)$, and $t_{1}$ and $m$ for $\boldsymbol{q} \propto(1,1,1)$. Here, $s$ denotes the mixture of the modes $t_{1}$ and $t_{2}$ that is given in the example above, and $m$ indicates mixtures of longitudinal and transverse modes [17]. Measurements of these modes are shown for several volume fractions in Fig. 1(b), where the lines represent a fit with general FCs of an fcc lattice with interactions up to third nearest neighbors. Three FCs $\left(A_{11}^{(1)}, A_{12}^{(1)}, A_{33}^{(1)}\right)$ are needed for the forces between nearest neighbors, while two $\left(A_{11}^{(2)}, A_{22}^{(2)}\right)$ and four $\left(A_{11}^{(3)}, A_{12}^{(3)}, A_{13}^{(3)}, A_{22}^{(3)}\right)$ are needed for the 2 nd and $3 \mathrm{rd}$ neighbors, respectively [2]. In Fig. 1(c), the 1st and 2nd neighbor FCs obtained from the fits are shown as a function of $\phi$. The absolute values of the third order constants $A_{\mu \nu}^{(3)}$ are found to be $\sim 0.01\left|A_{11}^{(1)}\right|$ and are not shown in the plot; they are not essential for the quality of the fits. For central forces, the FCs fulfill the condition $\Gamma:=A_{12}^{(1)} /\left(A_{11}^{(1)}-\right.$ $\left.A_{33}^{(1)}\right)=1$. However, from the measurements we find $\Gamma<$ 0.25 at all $\phi$. This is clearly incompatible with a pairwise additive, radially symmetric interaction. Furthermore, we observe $A_{11}^{(2)} \approx-2 / 3 A_{11}^{(1)}$, which means that a displacement of the particle at $(0,0,0)$ in the $(1,0,0)$ direction strongly attracts the 2 nd neighbor at $(a, 0,0)$. This also contradicts a HSY-like interaction.

To test the reliability of our data analysis and to exclude potential error sources, we performed MC simulations in the crystalline phase using the HSY-potential for the liquid as given above. We find that our data analysis yields FCs in perfect agreement with those calculated from the potential according to their definition. This is illustrated in Fig. 2(a), where crosses and lines represent the simulation and the $a b$ initio calculated band structure, respectively. The lower resolution of the microscope parallel to its optical axis has already been excluded as an error source by not using the corresponding component of $\boldsymbol{u}(\boldsymbol{q})$. However, there are further potential sources of errors: the time-lag between hexagonal layers due to the limited speed of the confocal scanner, finite-size effects, the time average used to calcu-

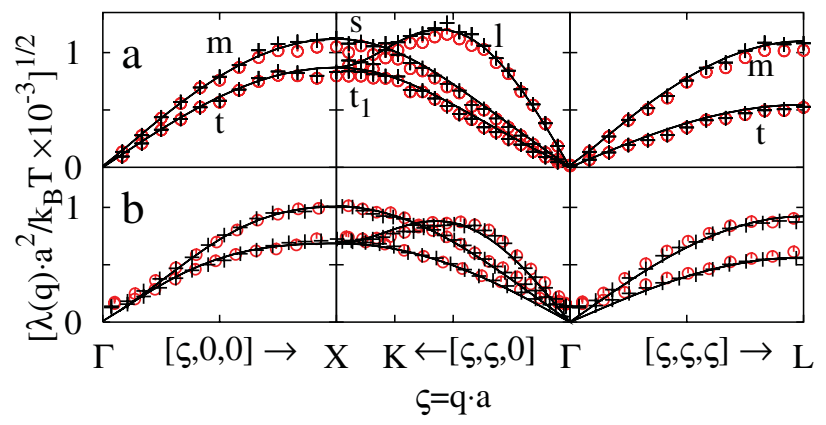

FIG. 2 (color). (a) Simulation: Effect of random errors $\Delta u$ added to the particle positions of a MC simulation with $\phi=$ 0.33, $B=15 k_{B} T$, and $\kappa \sigma=8$. Circles: $\Delta u \sim 0.02 \sigma$; crosses: no errors added. The lines represent the calculation. See text for details. (b) Experiment: Negligible finite-size effect in the measurements. The dispersion curves obtained from all (crosses) and from a subset (circles) of the particles in a measurement at $\phi=$ 0.31 are shown for comparison. The lines represent the best fit to the data. See text for details.

late the ensemble average in Eq. (1), and random errors in the particle coordinates. These are discussed below. The deviations of the particles that are used for the calculation of $\left\langle\left|u_{\mu}(\boldsymbol{q})\right|^{2}\right\rangle$ are not measured at strictly the same time. The scanning speed in the direction of the optical axis is limited by the exposure time $\approx 0.023 \mathrm{~s}$ of the CCD camera. It takes $0.12 \mathrm{~s}$ for the scanner to move from one hexagonal layer to the next and, during this time lag, the particles diffuse over a distance $\Delta r \approx 0.024 \sigma(\phi=0.31)$. This movement reduces the correlations between particles in different layers and, therefore, reduces the precision with which $u_{p}(\boldsymbol{q})$, the central quantity for determining the dispersion relations, can be determined. We modeled this effect by adding random errors of mean size $\Delta r$ to the coordinates of one particle in all terms of $\left\langle\left|u_{p}(\boldsymbol{q})\right|^{2}\right\rangle$ that involve particles from different hexagonal layers. It turns out that the effect of the time lag on the dispersion curves can be neglected. That a sufficiently large volume is observed, is illustrated in Fig. 2(b) where the measured dispersion curves of a crystal with $\phi=0.31$ containing 2090 particles in six hexagonal layers (crosses) are compared with those obtained from a subset of the same measurement containing only 592 particles in three layers (circles). Although the noise in the curves increases due to the reduced statistics, the curves overlap nicely. Therefore, we conclude that finite-size effects do not limit the accuracy of our measurements. By comparing the dispersion relations obtained from various numbers of particle configurations, we checked that a number of $\approx 70$ configurations is sufficient to obtain the dispersion curves. Since the measurements contained between 100 and 500 snapshots, the time average for approximating the ensemble average in Eq. (1) does not limit the accuracy of the dispersion relations. Random errors in the particle coordinates have the strongest effect on the dispersion curves. Figure 2(a) shows dispersion relations that are obtained from a MC 
simulation of 10032 particles with $\phi=0.33, B=15 k_{B} T$, and $\kappa \sigma=8$ with random, normally distributed errors added to the coordinates. When the width of the error distribution is set to $0.02 \sigma$, the correlations between the particle coordinates are still accurate enough to obtain a dispersion curve (circles) that is in good agreement with the direct calculation of the modes from the potential (lines) and the curve obtained without added errors (crosses). The strongest effect of these errors is found at $q$ values close to the boundary of the Brillouin zone, where the correlations between nearest neighbors are important. The importance of the positional errors in the measurements is least significant at low $\phi$, where the particles are relatively far apart. The $20 \mathrm{~nm}$ errors in the measured coordinates are below $0.02 \sigma \approx 30 \mathrm{~nm}$, which would still give a dispersion relation that is close to the true one.

We take the exclusion of the mentioned potential error sources as strong evidence for the significance of the determined FCs shown in Fig. 1(c). Therefore, we can determine all elastic constants of a colloidal crystal from a single measurement. From $\lim _{q \rightarrow 0} D(\boldsymbol{q}) / q^{2}$ we obtain the propagation coefficients for sound, which are directly related to the tensor $B_{\mu \nu \rho \sigma}$ connecting stress and strain: $T_{\mu \nu}=B_{\mu \nu \rho \sigma} \epsilon_{\rho \sigma}[1,18]$. For crystals under stress, $B_{\mu \nu \rho \sigma}$ is not the same as the tensor of elastic constants $C_{\mu \nu \rho \sigma}$, which is defined via the elastic free energy [1]. For the studied crystals $T_{\mu \nu}=-p \delta_{\mu \nu}$, as they are under stress due to the pressure $p$ in the sample cell; for fcc symmetry $B_{\mu \nu \rho \sigma}$ and $C_{\mu \nu \rho \sigma}$ are connected by the relations $B_{11}=$ $C_{11}-p, B_{12}=C_{12}+p$, and $B_{44}=C_{44}-p$ with the indices given in Voigt notation [1]. For central forces and cubic lattices, the Cauchy relation takes the form $B_{12}=$ $B_{44}+2 p$ for a crystal under pressure $p$. From the FCs at $\phi=0.31$ [Fig. 1(c)] we find $b_{11}=28, b_{12}=9$, and $b_{44}=$ 28 , where $b_{\mu \nu}=B_{\mu \nu} \sigma^{3} / k_{B} T$. Since $b_{44}>b_{12}$ and $p>0$, our measurements clearly contradict the Cauchy relation. Further, we obtain the bulk modulus $k:=\left(b_{11}+\right.$ $\left.2 b_{12}\right) / 3=15$ and the shear moduli $g_{1}:=b_{11}-b_{12}=$ 19 and $g_{2}:=b_{44}=28$. The elastic constants of HSY crystals from MC simulations fulfill the Cauchy relation. With the HSY potential given by $B=15 k_{B} T$ and $\kappa \sigma=8$ we obtain the shear moduli $g_{1}^{\mathrm{HSY}}=15$ and $g_{2}^{\mathrm{HSY}}=25$ that are comparable to those from experiment. The bulk modulus $k^{\mathrm{HSY}}=53$ is, however, more than 3 times larger; this is reflected by the large difference between the longitudinal $(\zeta, \zeta, 0)$ modes obtained from experiment [Fig. 2(b)] and simulation [Fig. 2(a)]. Thus, the noncentrality of the forces affects mostly the bulk modulus, which is also the case in metals.

Our main result is the clear demonstration of the incompatibility of the FCs with any effective radially symmetric pair potential and the importance of many-body forces. The distribution of the microions cannot be adequately approximated by a linear superposition of distributions around isolated colloids. As a consequence, also the forces between the colloids cannot be obtained from a superpo- sition of pairwise forces. Our results imply that the interaction between colloids at nearest neighbor distance is rather central in nature, while many-body forces dominate the interaction of a particle with its second nearest neighbors. This is reflected by the signs of $A_{33}^{(1)}$ as well as $A_{11}^{(2)}$ and $A_{22}^{(2)}$, which have the same and the opposite sign as expected for central forces, respectively. In the liquid it is hard to determine whether the forces are noncentral, since information about interactions and structural correlations is not easily separated [6]. However, as we have shown here, much less ambiguous results are obtained with crystals. In high symmetry directions, the eigenvectors of the dynamical matrix are determined by the lattice, while information about the interactions is contained exclusively in the eigenvalues, which determine the FCs. We conclude that the elastic properties and the behavior of the lattice normal modes in the studied colloidal crystals are rather metal- than HSY-like.

This work has been supported by the Deutsche Forschungsgemeinschaft (DFG) through subproject No. C4 of the SFB TR6 program.

[1] D.C. Wallace, in Solid State Physics, edited by H. Ehrenreich, F. Seitz, and D. Turnbull (Academic Press, New York, 1970), Vol. 25, pp. 301-404.

[2] P. Brüesch, Phonons: Theory and Experiments I (SpringerVerlag, Berlin, Heidelberg, New York, 1982).

[3] P. Pieranski, Contemp. Phys. 24, 25 (1983).

[4] B. Derjaguin and L. Landau, Acta Physicochim. URSS 14, 633 (1941).

[5] A.-P. Hynninen, M. Dijkstra, and R. van Roij, J. Phys. Condens. Matter 15, S3549 (2003); C. Russ et al., Phys. Rev. E 66, 011402 (2002); J. Dobnikar, R. Rzehak, and H.-H. von Grünberg, Europhys. Lett. 61, 695 (2003).

[6] M. Brunner et al., Europhys. Lett. 58, 926 (2002).

[7] M. Brunner et al., Phys. Rev. Lett. 92, 078301 (2004).

[8] C. Russ et al., Europhys. Lett. 69, 468 (2005).

[9] J. A. Weiss, A. E. Larsen, and D. G. Grier, J. Chem. Phys. 109, 8659 (1998).

[10] A. J. Hurd et al., Phys. Rev. A 26, 2869 (1982); Z. D. Cheng et al., Phys. Rev. Lett. 85, 1460 (2000).

[11] L. Antl et al., Colloids Surf. 17, 67 (1986).

[12] J. C. Crocker and D. G. Grier, J. Colloid Interface Sci. 179, 298 (1996).

[13] A.-P. Hynninen and M. Dijkstra, Phys. Rev. E 68, 021407 (2003).

[14] C.-P. Royall, M.-E. Leunissen, and A. van Blaaderen, J. Phys. Condens. Matter 15, S3581 (2003).

[15] P. N. Pusey et al., Phys. Rev. Lett. 63, 2753 (1989).

[16] P. Keim et al., Phys. Rev. Lett. 92, 215504 (2004).

[17] $s$-mode:, e.g., $\hat{\boldsymbol{e}}_{s}=1 / \sqrt{6}(-1,-1,2)$ for $\boldsymbol{q} \propto(1,-1,0)$; $m$-modes:, e.g., $\hat{\boldsymbol{e}}_{m}=1 / \sqrt{6}(2,-1,-1)$ for $\boldsymbol{q} \propto(1,0,0)$ and, e.g., $\hat{\boldsymbol{e}}_{m}=1 / \sqrt{6}(1,1,-2)$ for $\boldsymbol{q} \propto(1,1,-1)$.

[18] $B_{11}=\frac{a^{2}}{v_{0}}\left[-A_{11}^{(1)}-A_{11}^{(2)}\right], \quad B_{12}=\frac{a^{2}}{2 v_{p}}\left[-2 A_{12}^{(1)}+A_{11}^{(1)}+\right.$ $\left.A_{33}^{(1)}+{ }_{2}^{v_{0}} A_{22}^{(2)}\right]$, and $B_{44}=\frac{a^{2}}{2 v_{0}}\left[-A_{11}^{(1)}-\stackrel{2}{(\rho 1)}_{33}-2 A_{22}^{(2)}\right] . v_{0}$ denotes the volume of the primitive cell. 